\title{
A meta-analysis of clinical efficacy and quality of life of cognitive-behavioral therapy in acute coronary syndrome patients with anxiety and depression
}

\author{
Hang $\mathrm{Yu}^{1}$, Yunyan $\mathrm{Ma}^{2}$, Rong $\mathrm{Lei}^{3}$, Dan $\mathrm{Xu}^{4}$ \\ ${ }^{1}$ Department of Cadre Health Care, ${ }^{2}$ Blood Purification Center, ${ }^{3}$ Health Management Center, Affiliated Hospital of North Sichuan Medical College, \\ Nanchong, China; ${ }^{4}$ Department of Nephrology, the First Affiliated Hospital of Chengdu Medical College, Chengdu, China \\ Contributions: (I) Conception and design: H Yu, D Xu; (II) Administrative support: Y Ma, D Xu; (III) Provision of study materials or patients: H Yu, \\ Y Ma, R Lei; (IV) Collection and assembly of data: All authors; (V) Data analysis and interpretation: H Yu, R Lei, D Xu; (VI) Manuscript writing: All \\ authors; (VII) Final approval of manuscript: All authors. \\ Correspondence to: Dan Xu. Department of Nephrology, the First Affiliated Hospital of Chengdu Medical College, 278 Baoguang Avenue, Xindu \\ District, Chengdu, China. Email: xudanxddd@163.com.
}

Background: Our study aims to evaluate the clinical efficacy and quality of life of cognitive-behavioral therapy (CBT) for patients who have acute coronary syndrome (ACS) with anxiety and depression.

Methods: The databases of PubMed, Cochrane, and Web of science, as well as China journal full-text database, Wanfang database, and Weipu database, were systematically searched from the establishment of the database to February 29, 2020. The total effective rate of qualitative data was evaluated with a relative risk (RR) and 95\% confidence interval (CI), and the quantitative data was evaluated with a standard mean difference (SMD) and 95\% CI. Randomized controlled clinical trials of CBT for ACS were included. The RevMan5.3 and R3.5.1 software was used to analyze.

Results: A total of 11 papers, including 1,259 patients, were included, including 639 patients in the CBT group and 620 in the control group. One article reported the total effective rate after three months of treatment with an RR of 1.48 (95\% CI: 1.07, 2.06). A total of 3 articles reported the incidence of cardiovascular adverse events using the fixed effects model $\left(\mathrm{I}^{2}=41 \%\right)$, and the incidence of cardiovascular adverse events in the CBT group was 0.39 times lower than that in the control group (95\% CI: 0.2, 0.77). A total of 11 articles reported the score of depression using the random effects model $\left(\mathrm{I}^{2}=93 \%\right)$, and the score of depression in the CBT group was significantly lower than that in the control group, with an SMD of -0.99 (95\% CI: -1.44, -0.54). The score of anxiety was reported in 8 pieces of literature, and the randomized effect model estimated that the score of anxiety in the CBT group was significantly lower than that in the control group, with an SMD of -1.47 (95\% CI: -1.98, -0.96). There was significant heterogeneity in the quality of life score, but it was not found that the quality of life score in the CBT group was significantly higher than that in the control group.

Conclusions: After CBT intervention, ACS patients with anxiety and depression can significantly reduce the incidence of cardiovascular adverse events and significantly reduce scores of depression and anxiety, but they have not been found to have an advantage in improving quality of life.

Keywords: Acute coronary syndrome (ACS); anxiety and depression; cognitive-behavioral therapy (CBT); metaanalysis

Submitted Mar 23, 2020. Accepted for publication May 27, 2020.

doi: 10.21037/apm-20-974

View this article at: http://dx.doi.org/10.21037/apm-20-974 


\section{Introduction}

Acute coronary syndrome (ACS) is a high-risk disease with acute onset, severe illness, rapid changes, and high mortality and disability (1). There are three clinical types of ACS: ST-segment elevation myocardial infarction, nonST-segment elevation myocardial infarction, and unstable angina. The treatment methods for different types of ACS are different. However, ACS treatment mainly includes anti-thrombotic therapy, anti-myocardial ischemic therapy, stable plaque therapy, interventional therapy, and coronary artery bypass and others (2). Due to the high cost of medical treatment and the need for repeated follow-up visits and long-term medication for patients with ACS, patients suffer from a heavy financial burden and great distress, which brings negative emotions such as anxiety and depression for the patients (3). Anxiety and depression have public health significance, but most patients with anxiety and depression are not detected and treated by the medical system in time. Studies have shown that depression and anxiety increase the mortality of ACS patients and the incidence of cardiovascular events, which seriously affect the prognosis and life quality of patients (4). ACS interacts with anxiety and depression. Patients with anxiety and depression respond poorly to the treatment and often have a poor prognosis. A coronary psychosocial evaluation study was conducted on ACS patients with persistent depression. The results show that active treatment of depression can reduce the risk of death or rehospitalization of myocardial infarction or unstable angina (5). Cognitivebehavioral therapy (CBT) has gradually been applied to ACS treatment with the emphasis on the "bio-psycho-social" medical model (6). As an effective method of psychological intervention, CBT corrects patients' misunderstanding and irrational cognition through verbal and non-verbal communication, thereby changing the bad psychological state and wrong behavior, establishing correct cognition, and elevating the courage of patients to face reality. It can help to reduce the physical and psychological pain of patients, and thus promote patient recovery (7). A metaanalysis evaluated the safety and effectiveness of rational emotional behavioral therapy for the past 50 years. Rational emotional behavioral therapy is a type of CBT. By reviewing the 68 included literatures, it is found that compared with other interventions and irrational faith, rational emotional behavior therapy has a better effect (8).

With the advent of global aging and the accelerated pace of life, the influence from anxiety and depression have become more serious. And in China, the incidence, mortality, and readmission rates of ACS all show an increasing trend year by year (9). Good mental health and social support can significantly improve the life quality of ACS patients and improve long-term prognosis. It is still controversial about the clinical efficacy and long-term outcome in the current literature on the treatment of CBT in ACS patients with anxiety and depression due to the small sample size and the difference of intervention content. At present, there is no literature on a comprehensive and objective evaluation of CBT in ACS patients with anxiety and depression. Therefore, in this study, a meta-analysis method was used to comprehensively evaluate the above issues, aiming to provide the best evidence of evidencebased medicine for CBT of ACS and guide clinical treatment.

We present the following article in accordance with the PRISMA reporting checklist (available at http://dx.doi. org/10.21037/apm-20-974).

\section{Methods}

\section{Literature search}

English databases were systematically search including PubMed, Cochrane and Web of science, and keywords and subject words were combined for retrieval.: "acute coronary syndrome", "acute coronary artery syndrome", "ACS" and "Depression”, "depressed", "despondent", "gloomy"; keywords of interventions: "cognitive behavioral therapy", "Cognitive-behavioral intervention", "cognitive activity", "Cognitive actions", "cognitive-behavioral", "cognitive behavior", "CBT". Keywords of patient and intervention related to AND. The Chinese databases were searched, including the Chinese Journal Full-text Database (CNKI), Wanfang Database, and Weipu Database. The keywords were combined with "acute coronary syndrome", "acute coronary syndrome" and "anxiety", "depression", and "cognition behavior", "cognitive therapy", "behavioral therapy." The retrieval time was from the establishment of the database to February 29, 2020. At the same time, the references included in the literature were retrieved through Google Scholar and others.

\section{Inclusion and exclusion criteria}

Literature that met the following criteria were included in this analysis: (I) all patients are with ACS disease met 
the ACC/AHA or clinical diagnostic criteria; (II) patients in the control group are treated with usual treatments such as antiplatelet aggregation, lipid regulation, coronary expansion, nourishing myocardium and improving circulation treatment or routine care and received routine health education; (III) patients in the experimental group are added cognitive-behavioral interventions on the basis of control, including comprehensive intervention, doubleheart intervention, psychological intervention, etc.; (IV) the trial is designed as a randomized clinical controlled trial (RCT); (V) the outcome indicators include any of the following index: total effectiveness rate, incidence of adverse cardiovascular events, score of depression before and after treatment including the self-depression rating scale (SDS) and depression scale (PHQ-9), score of anxiety before and after treatment including self-rating anxiety scale (SAS) and self-rating anxiety scale (GAD-7), score of quality of life before and after treatment including physical activity limitation (PL) of the Seattle angina pectoris scale (SAQ), anginal stability (AS), angina pectoris (AF), treatment satisfaction (TS), DS, short form 36 health survey (SF-36); (VI) outcome measures are admission or discharge, and treatment for 3 or 6 months. The evaluation criteria for anxiety and depression were as follows: the lower the difference between the two groups before and after treatment, the better the improvement effect of anxiety and depression. But for the quality of life score comparison before and after treatment, the higher the value, the more significant improvement in quality of life.

Exclusion criteria: (I) ACS patients suffer from bipolar disorder, cerebral organic mental disorder or inability to communicate; (II) animal experiments, reviews, case reports, comments, abstracts or other non-research article forms; (III) data are measured 24 hours after surgery; (IV) literature is unable to extract data; (V) duplicate publications and the most comprehensive publication data.

\section{Literature screening and data extraction}

Two data analysts independently read the title, abstract, and full text conducted literature screening according to the inclusion and exclusion criteria and then used a unified data extraction form for data extraction and verification of the literature that met the inclusion and exclusion criteria. The data extracted from the literature included: author, publication date, age and gender of the research subjects, therapies, sample size, number of effective and adverse events, the mean and standard deviation of anxiety and depression cases in the control group and the experimental group. Where there is an inconsistency between literature screening and data extraction, a third person will review it.

\section{Evaluation of literature quality}

Literature included in this study conducted with RCT. Therefore, the Cochrane risk bias assessment tool (10) was used to evaluate the risk of bias mainly from six areas: selection bias, implementation bias, measurement bias, follow-up bias, reporting bias and other biases. Each index was evaluated as low, unclear, and high bias.

\section{Statistical analysis}

Effect indicators of effectiveness and adverse cardiovascular events were indicated using ratio risk (RR) and $95 \%$ confidence interval (CI); depression and anxiety were evaluated with a standard mean difference (SMD) and 95\% CI. $\mathrm{I}^{2}$ was used for heterogeneity test. When $\mathrm{I}^{2} \leq 50 \%$, a fixed-effect model was used, while when $\mathrm{I}^{2}>50 \%$, a randomeffect model was used for an effective combination. For heterogeneous research, the research objects were divided into subgroups of China and the United States to explore the source of heterogeneity. And sensitivity analysis was used to explore whether the existence of a document has a significant impact on the whole results. Begg rank correlation and Egger regression were used to quantifying the effect of publication indexes with more than ten articles. Quality evaluation of literature and data analysis were performed using Revman 5.3 and R 3.5.1 software for statistical analysis.

\section{Results}

\section{Basic information of included literature}

A total of 105 Chinese and English literatures were retrieved. According to the exclusion criteria, 11 pieces of literature were finally included, with a total of 1,259 ACS patients in the RCT. There were four different papers $(11-14)$ and 7 Chinese papers $(7,15-20)$ among the entire 11 pieces of literature, a total of 639 patients in the experimental group were using CBT, and 620 patients in the control group received conventional treatment or care. The process of the literature search was shown in Figure 1, and the necessary information of the included literature was shown in Table 1. 


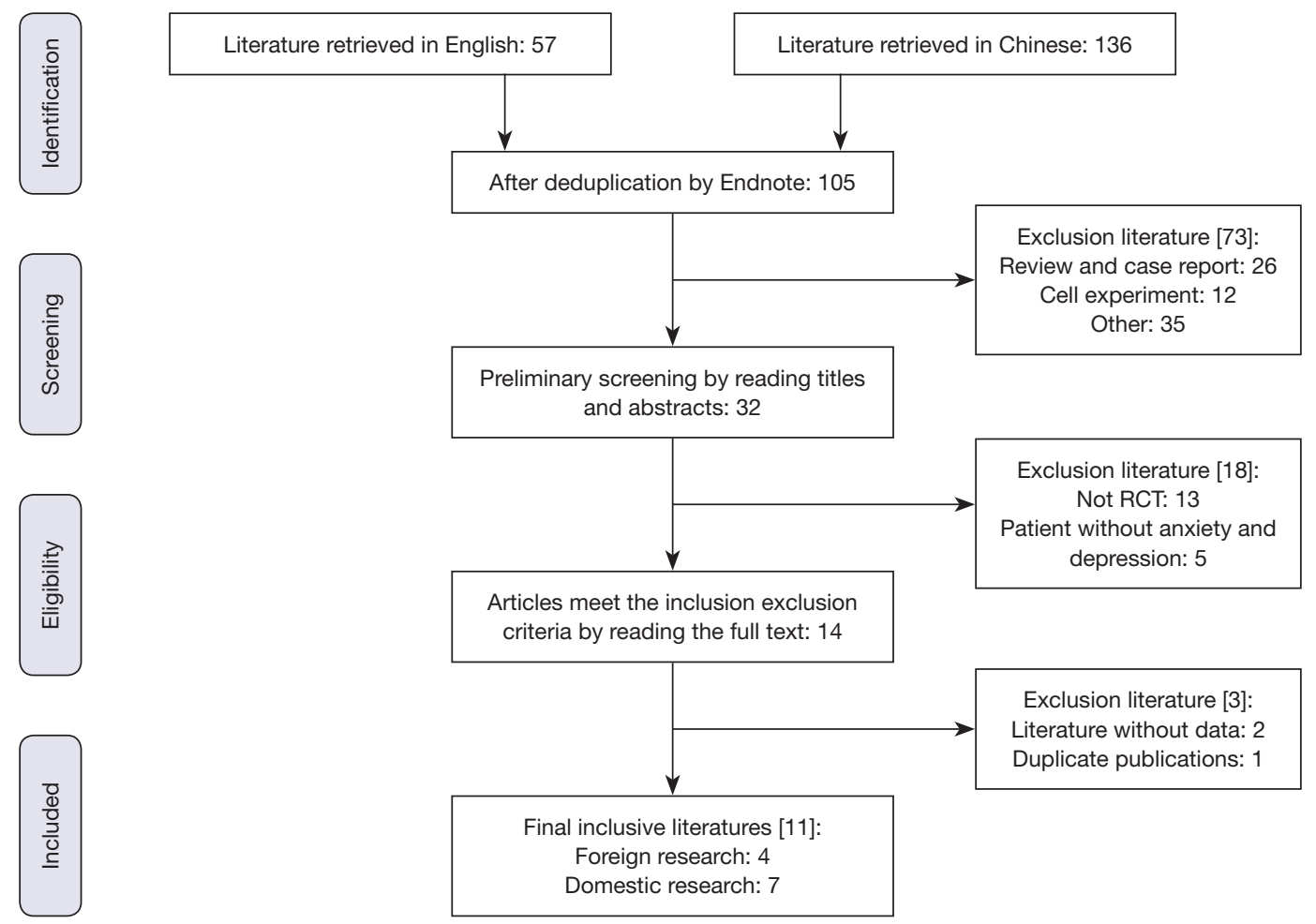

Figure 1 Flow chart of literature retrieval.

\section{Literature quality evaluation}

Among the included 11 pieces of literature, patients were all randomly grouped, but only one article (17) did not explain what random method was used. Using random grouping can avoid patient selection bias, but implementation bias was not explained clearly in these articles. Follow-up bias, report bias, and bias of other aspects were at minimal risk. Cochrane risk bias assessments were shown in Figure 2.

\section{Meta-analysis of the effective rate}

Only one article (19) reported the total effective rate after three months of treatment, with an RR value of 1.48 , which means that the total effective rate of the experimental group with CBP was 1.48 times (95\% CI: 1.07, 2.06) higher than the control group.

\section{Meta-analysis of the cardiovascular adverse event}

A total of 3 pieces of literature $(7,15,19)$ reported the incidence of cardiovascular adverse events $\left(I^{2}=41 \%\right)$ after 3 or 6 months of treatment. The RR was estimated using a fixed-effect model to find that the incidence of adverse reactions in the experimental group was 0.39 times ( $95 \%$ CI: $0.2,0.77)$ lower than that of the control group, as shown in Figure 3.

\section{Meta-analysis of the depression score}

A score of depression after treatment was reported in a total of 11 pieces of literature. Because some pieces of literature used SDS, and some others used PHQ-9, SMD was used to combine the effects. The overall heterogeneity was high $\left(\mathrm{I}^{2}=93 \%\right)$, so a random effect model was used to assess SMD. The score of depression in the experimental group was significantly lower than that in the control group, with an SMD of -0.99 (95\% CI: $-1.44,-0.54)$. After stratified analysis, according to different countries, the heterogeneity decreased. In the United States, the score of depression in the experimental group was lower than that in the control group, with an SMD of -0.29 (95\% CI: -0.58, 0). In China, the score of depression in the experimental group was lower than that in the control group, with an SMD of -1.40 (95\% CI: $-1.98,-0.81)$, where the decline was higher than that in the United States, as shown in Figure 4. 
Table 1 Basic information of the included literature of CBT for ACS patients with anxiety and depression

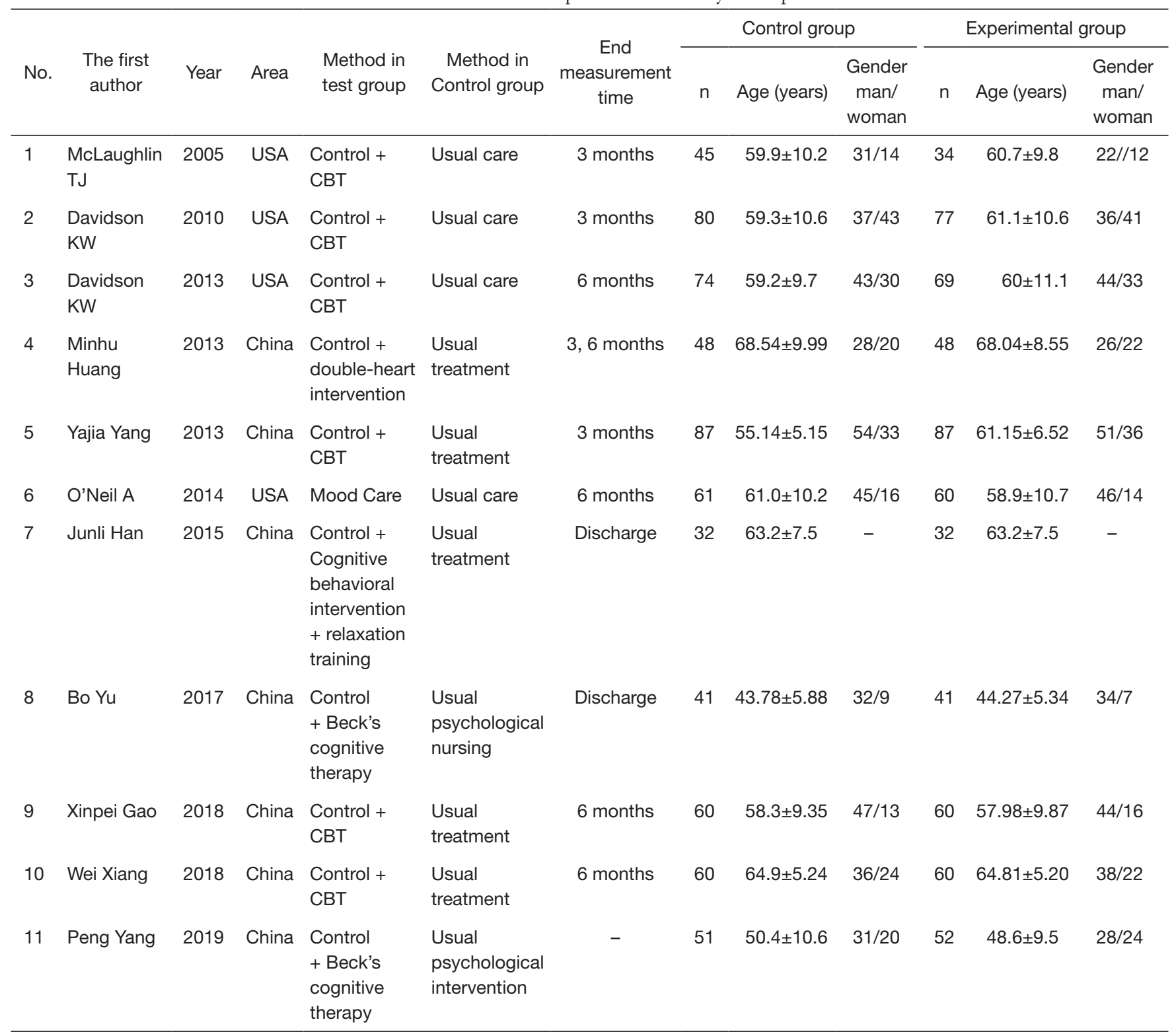

CBT, cognitive-behavioral therapy; ACS, acute coronary syndrome.

\section{Meta-analysis of the anxiety score}

There were eight articles reported the score of anxiety after treatment, so SMD was used for assessment of effect combination, which indicated that the overall heterogeneity was high $\left(\mathrm{I}^{2}=90 \%\right)$. The random-effect model was used to estimate SMD. The result showed that the score of anxiety in the experimental group was significantly lower than that in the control group, with an SMD of -1.47 (95\% CI: -1.98, -0.96). After the stratified analysis according to different countries, apart from only one article in the United States, the score of anxiety in the experimental group is lower than that in the control group in China, with an SMD of -1.57 (95\% CI: -2.13, -1.01), as shown in Figure 5.

\section{Meta-analysis of the quality of life score}

There was a more heterogeneous quality of life score, but none of them were found that the quality of life score in the CBT group was significantly higher than that in the control 
group after treatment, as shown in Table 2.

\section{Evaluation of sensitivity and publication bias}

The sensitivity analysis was performed after excluding the literature one by one, and it was found that the research

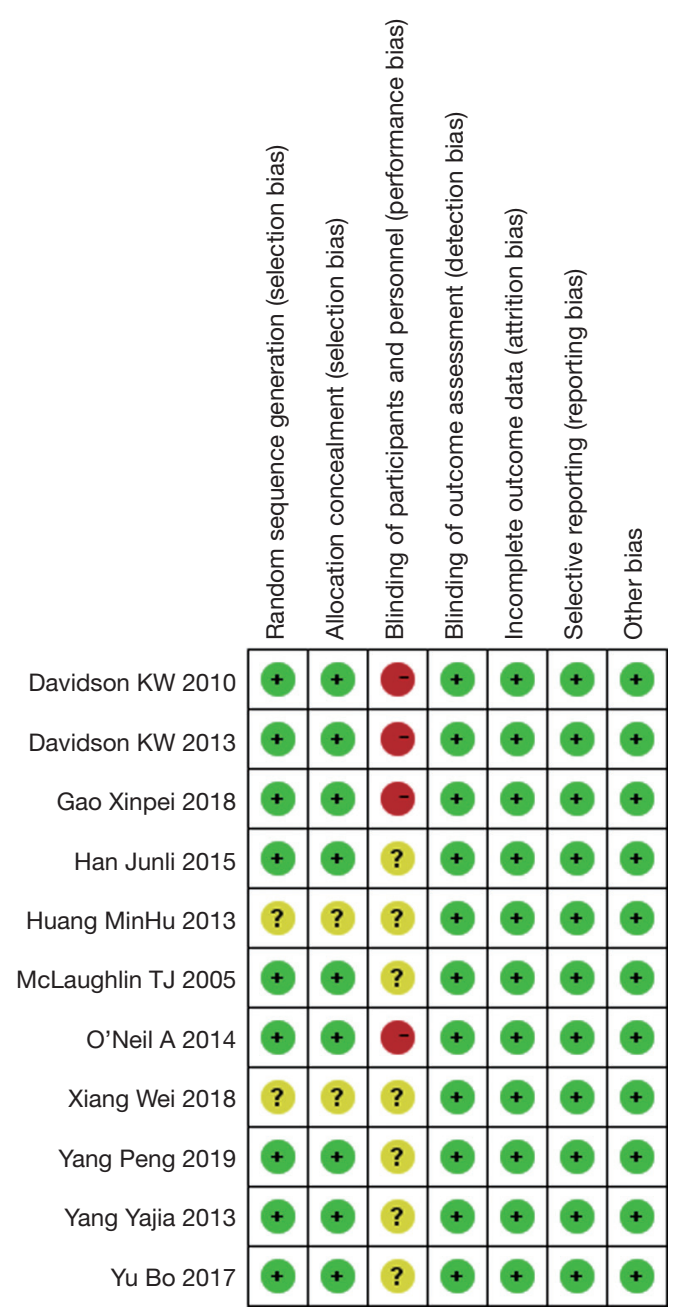

Figure 2 Evaluation of quality bias in the included literature. results were stable and showed no significant change. After Egger regression and Begg rank correlation analysis, there was a publication bias in the scores of depression and anxiety $(\mathrm{P}<0.05)$, as shown in Table 3.

\section{Discussion}

In this study, a meta-analysis was firstly used to reveal that the incidence of cardiovascular adverse events in ACS patients with anxiety and depression after CBT intervention was significantly lower than that in the control group receiving the conventional treatment or care, and the incidence of adverse events was 0.39 times lower than that in the control group. The CBT intervention could effectively reduce the scores of depression and anxiety in ACS patients, but compared with the control group, and there was no significant difference in the quality of life of ACS patients in the test group, which may be related to the small number of included literatures. Since only one article reported the efficacy after treatment, a comprehensive evaluation of the effective rate could not be obtained right now.

CBT is widely used in nursing, clinical psychology and other fields, which is a combination of cognitive theory and behavior therapy, where the cognitive behavior theory is integrated from cognitive theory and behavior theory. An important part of cognitive theory is "ABC Emotion Theory Framework": A (Activating event), B (Belief) and C (Consequence). The main point of the cognitive behavior theory is that the relationships of cognition, behavior, and emotion are not separated from each other, but inseparable, influential, and interrelated to each other. The treatment principle of CBT attaches importance to the correction of internal cognition and emphasizes the importance of structural interviews. During the treatment process, patients should be taught to identify, debate, reshape their thinking and beliefs, and use different techniques to help patients change their cognition, behaviors and emotions; In addition, if the patients themselves can recognize and

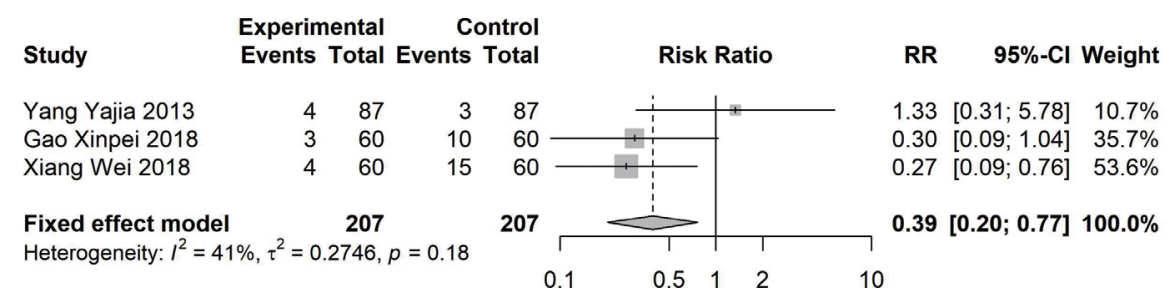

Figure 3 Forest plot of meta-analysis of cardiovascular adverse events of CBT in ACS patients with anxiety and depression. CBT, cognitivebehavioral therapy; ACS, acute coronary syndrome. 


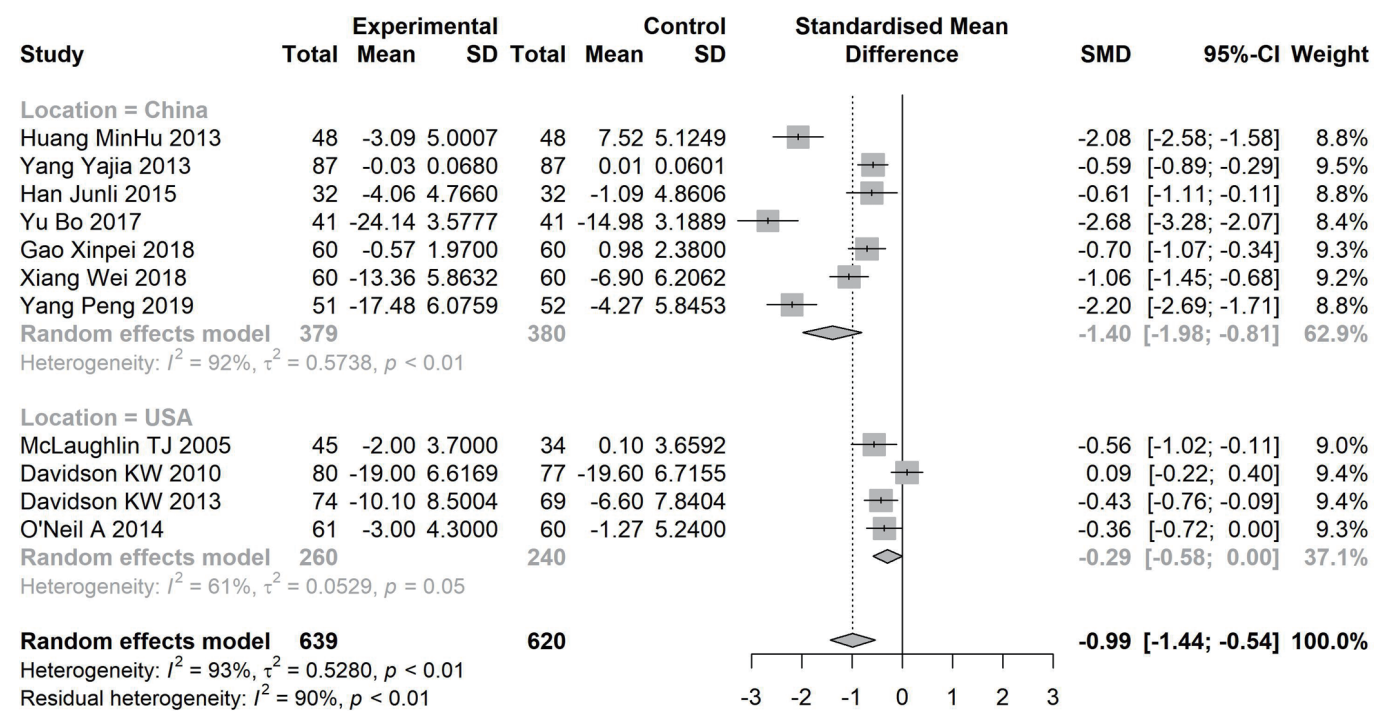

Figure 4 Forest plot of meta-analysis of depression score of CBT in ACS patients with anxiety depression. CBT, cognitive-behavioral therapy; ACS, acute coronary syndrome.

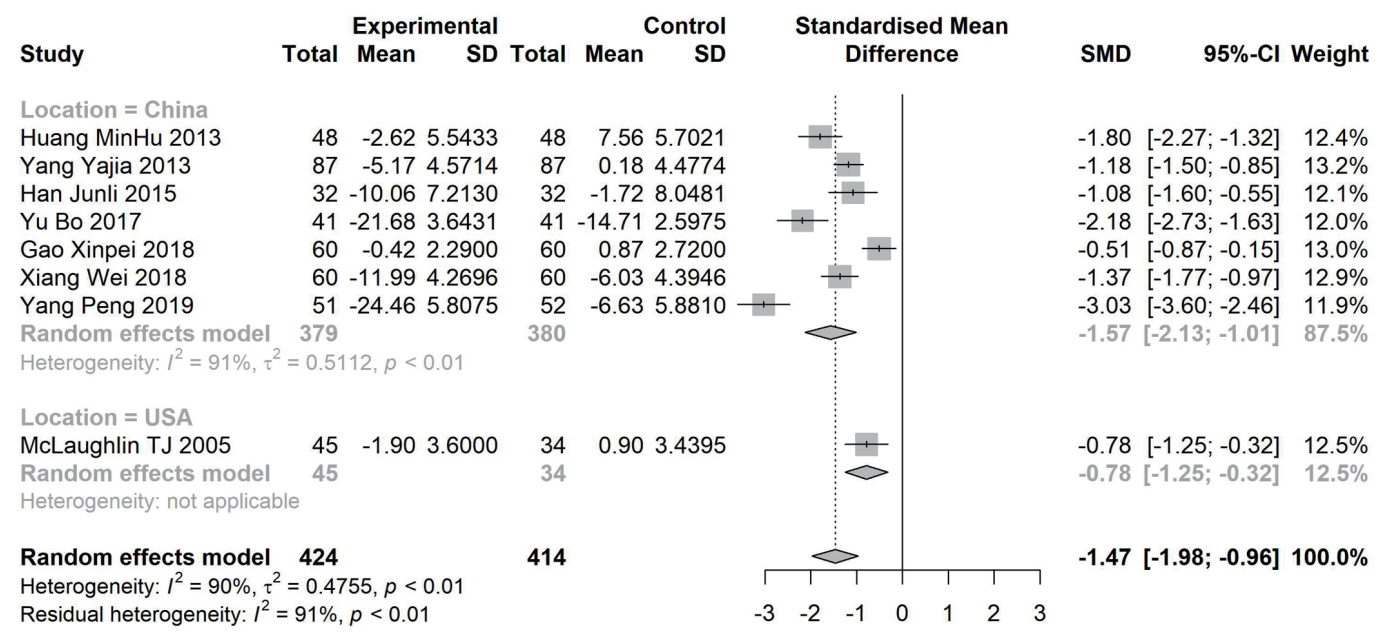

Figure 5 Forest plot of meta-analysis of anxiety score of CBT in ACS patients with anxiety and depression. CBT, cognitive-behavioral therapy; ACS, acute coronary syndrome.

change irrational beliefs and achieve self-control after the treatment, the patients' power is enhanced (21).

Patients with ACS often develop depression and fear inevitably. With CBT intervention, the patient's cognition can be improved due to the adjustment of mentality (14). For anxiety and depression, medical staff regularly correct misbehavior of patients with ACS, listen to their demands patiently, supply comfort and encouragement, and establish a good doctor-patient relationship. The patient's mental stress can be relieved through various forms of health preaching, step-by-step explanation targeting different educational levels of patients to help them correctly understand the disease-related risk factors, the causes, and harms of the disease. At the same time, through some comprehensive interventions, relax training and psychological treatment by health professionals to strengthen psychological hint for patients continually, so that patients maintain an optimistic and positive attitude, appropriately participate in social activities and appropriate physical exercise to promote circulation function, thereby 
Table 2 Meta-analysis of life quality scores of CBT in ACS patients with anxiety and depression

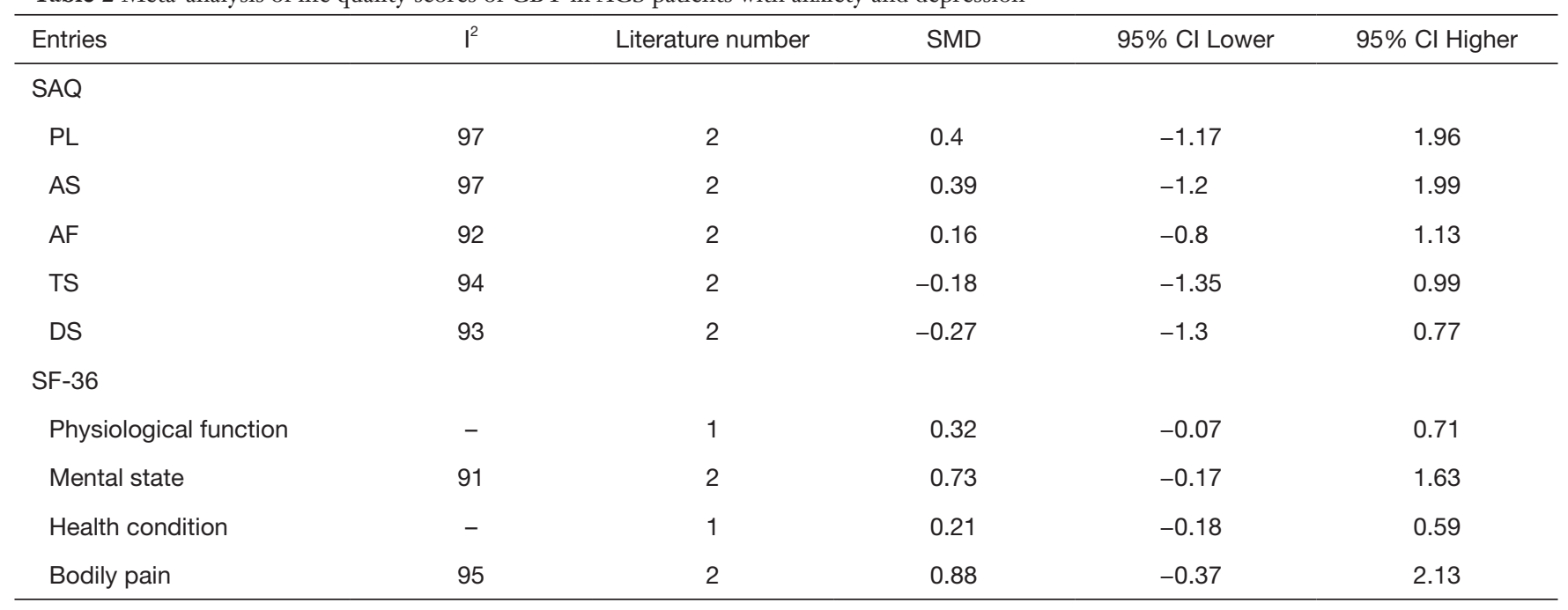

CBT, cognitive-behavioral therapy; ACS, acute coronary syndrome.

Table 3 Publication bias evaluation in a meta-analysis of CBT in ACS patients with anxiety and depression

\begin{tabular}{lccccc}
\hline \multirow{2}{*}{ Kinds } & \multicolumn{3}{c}{ Egger } & & Begg \\
\cline { 2 - 5 } & $\mathrm{t}$ & $\mathrm{P}$ & $\mathrm{Z}$ & $\mathrm{P}$ \\
\hline Depression & -3.934 & 0.003 & -2.569 & 0.010 \\
Anxiety & -2.058 & 0.085 & -2.227 & 0.026 \\
\hline
\end{tabular}

CBT, cognitive-behavioral therapy; ACS, acute coronary syndrome.

helping patients to improve their clinical efficacy (22-24).

Through meta-analysis, we found that the scores of anxiety and depression of patients were significantly reduced through CBT intervention, and the scores of anxiety and depression of patients with ACS after CBT intervention were significantly lower than those of the control group. The results of this study were not related to the area, population, and content of research, which fully demonstrates that psychological intervention-based treatment of CBT has a positive, stimulating effect on improving anxiety and depression of patients with ACS. It is indicated that anxiety and depression can trigger or aggravate diseases such as angina pectoris, myocardial infarction, arrhythmia and other diseases, which brings a substantial economic and disease burden to the whole society (25). Therefore, it can help to improve the prognosis of patients by improving the symptoms of anxiety and depression in patients, At the same time, the results of the meta-analysis found that the incidence of cardiovascular adverse events in ACS patients treated with CBT was significantly reduced, which further proved that the comprehensive intervention method of CBT could significantly improve the prognosis of patients. However, the quality of life score of patients treated with CBT was not significantly different from those of usual intervention. Nevertheless, we cannot conclude that the quality of life in ACS patients with CBT intervention is the same as that of usual intervention patients, because the metaanalysis of the quality of life score only included a few pieces of literature, suggesting that more studies need to be collected for further analysis.

The literatures included in this analysis are all RCT, and the quality of these kinds of literature was good. Ten pieces of literature are reporting random grouping methods, but the implementation bias is not explained, which is due to the difficulty of double-blindness of CBT intervention. There are four pieces of literature in the United States at high risk, but there is no explanation in the domestic research with definition as unclear risk. Although the quality of the literature in this study is good, the heterogeneity of the combined effect is still significant. Following the source of the research aims, the analysis was divided into the United States and China, respectively. This was done to determine that the heterogeneity was reduced. In China, the score of depression in the experimental group was lower than that in the control group, and the degree of decline was higher than that in the 
United States, with an SMD of -1.40 (95\% CI: $-1.98,-0.81$ ). Different kinds of literature use different questionnaires to score depression. For example, Huang uses the Hamilton depression scale (HAMD) (17), Gao and Davidson use PHQ-9 as the depression scale $(11,15)$, and in most kinds of literature, the score of depression is performed using SDS. Different scales have different scoring entries, different total scores, and different standards for judging depression, so the heterogeneity of the included analysis is high.

Meanwhile, it is also caused by that the methods of cognitive-behavioral interventions vary between different countries, the contents of the interventions are not the same, the measurement time after treatment is inconsistent including discharged, three months after discharge and followed-up for six months. All the differences in these factors are important reasons for the high heterogeneity. Due to the small number of research literature, it is impossible to do further stratified analysis. These problems also exist in the analysis of scores of anxiety and quality of life, leading to high heterogeneity.

The sensitivity study in this study found that the metaanalysis results of the incidence of adverse cardiovascular events, depression scores, anxiety scores, and quality of life scores were relatively stable, and no particular study was found to have a significant impact on the results. At the same time, the quantitative evaluation of Begg and Egger showed that there was a publication bias in this metaanalysis, but it is worth noting that when the number of articles is less than 20, the sensitivity of publication bias results is reduced. However, some negative results also exist even though they are difficult to publish.

The meta-analysis in this study has limitations. (I) The scope of the CBT methods is broad, and the content of the interventions are different, but they are all based on psychological intervention. (II) The research results are heterogeneous, except for the differences of regions, anxiety, depression, and quality of life and differences in the definitions of the indicators. The inconsistencies in the measurement time are also some of the reasons for high heterogeneity. However, due to the limited data, it is not yet possible to carry out the related stratified analysis. (III) The sample size included in this study is generally small, and the conclusion still needs to be confirmed in further research. (IV) Lack of sufficient literature to comprehensively analyze the efficacy evaluation of CBT intervention.

In summary, after CBT intervention in ACS patients with anxiety and depression, the incidence of cardiovascular adverse events, the scores of depression and anxiety are significantly reduced. However, it is not found to be an advantage in improving the quality of life. Due to the small sample size of the included pieces of literature and the lack of research on clinical efficacy, high-quality clinical studies of large samples are needed to do further analysis of clinical effectiveness and safety.

\section{Acknowledgments}

Funding: None.

\section{Footnote}

Reporting Checklist: The authors have completed the PRISMA reporting checklist. Available at http://dx.doi. org/10.21037/apm-20-974

Conflicts of Interest: All authors have completed the ICMJE uniform disclosure form (available at http://dx.doi. org/10.21037/apm-20-97). The authors have no conflicts of interest to declare.

Ethical Statement: The authors are accountable for all aspects of the work in ensuring that questions related to the accuracy or integrity of any part of the work are appropriately investigated and resolved.

Open Access Statement: This is an Open Access article distributed in accordance with the Creative Commons Attribution-NonCommercial-NoDerivs 4.0 International License (CC BY-NC-ND 4.0), which permits the noncommercial replication and distribution of the article with the strict proviso that no changes or edits are made and the original work is properly cited (including links to both the formal publication through the relevant DOI and the license). See: https://creativecommons.org/licenses/by-nc-nd/4.0/.

\section{References}

1. Pour-Ghaz I, Bob-Manuel T, Marella HK, et al. Incidence and predictors of acute coronary syndrome within a year following a negative stress test-a false sense of security: is routine screening any useful? Ann Transl Med 2018;6:13.

2. Kim C, Choi D. Timing of high intensity statin for acute coronary syndrome: how earlier initiation makes better? J Thorac Dis 2018;10:S2149-52.

3. Yuan J, Ding R, Wang L, et al. Screening for depression in acute coronary syndrome patients: A comparison of 
Patient Health Questionnaire-9 versus Hospital Anxiety and Depression Scale-Depression. J Psychosom Res 2019;121:24-8.

4. Xia K, Wang LF, Yang XC, et al. Comparing the effects of depression, anxiety, and comorbidity on quality-of-life, adverse outcomes, and medical expenditure in Chinese patients with acute coronary syndrome. Chin Med J (Engl) 2019;132:1045-52.

5. Ye S, Shaffer JA, Rieckmann N, et al. Long-term outcomes of enhanced depression treatment in patients with acute coronary syndromes. Am J Med, 2014;127:1012-6.

6. O'Neil A, Taylor B, Hare DL, et al. Long-term efficacy of a tele-health intervention for acute coronary syndrome patients with depression: 12-month results of the MoodCare randomized controlled trial. Eur J Prev Cardiol 2015;22:1111-20.

7. Xiang $\mathrm{W}$, Huang $\mathrm{YL}, \mathrm{Ma} \mathrm{B}$, et al. Influence of cognitive behavior intervention on quality of life and prognosis of ACS patients with anxiety and depression. Applied Journal of General Practice 2018;16:207-9, 304.

8. David D, Cotet C, Matu S, et al. 50 years of rationalemotive and cognitive-behavioral therapy: A systematic review and meta-analysis. J Clin Psychol 2018;74:304-18.

9. He P, Liu Y, Wei X, et al. Comparison of enoxaparin and unfractionated heparin in patients with non-STsegment elevation acute coronary syndrome undergoing percutaneous coronary intervention: a systematic review and meta-analysis. J Thorac Dis 2018;10:3308-18.

10. Higgins JPT, Green S. Cochrane Handbook for Systematic Reviews of Interventions Version 5.1.0(EB/OL). The Cochrane Collaboration, 2011 (2012-03-30). Available online: http:// www. Cochrane-handbook.org

11. Davidson KW, Bigger JT, Burg MM, et al. Centralized, stepped, patient preference-based treatment for patients with post-acute coronary syndrome depression: CODIACS vanguard randomized controlled trial. JAMA Intern Med 2013;173:997-1004.

12. Davidson KW, Rieckmann N, Clemow L, et al. Enhanced depression care for patients with acute coronary syndrome and persistent depressive symptoms: coronary psychosocial evaluation studies randomized controlled trial. Arch Intern Med 2010;170:600-8.

13. McLaughlin TJ, Aupont O, Bambauer KZ, et al. Improving psychologic adjustment to chronic illness in cardiac patients. The role of depression and anxiety. J Gen Intern Med 2005;20:1084-90.

14. O'Neil A, Taylor B, Sanderson K, et al. Efficacy and feasibility of a tele-health intervention for acute coronary syndrome patients with depression: results of the
"MoodCare" randomized controlled trial. Ann Behav Med, 2014;48:163-74.

15. Gao XP. Effect of the psycho-cardiology treatment on quality of life in patients with acute coronary syndrome. North China University of Science and Technology, 2018.

16. Han JL, Zeng BM, Chen J. Effect of cognitive behavior intervention on acute coronary syndrome patients with negative emotions. Jilin Medical Journal 2015;36:123-5.

17. Huang MH. Application research of psycho-cardiology treatment model in Acute Coronary Syndrome. North Sichuan Medical College, 2013.

18. Yang P, Zhou YY, Yu CC. Effect of psychological intervention based on Beck's cognitive therapy on mood and quality of life in patients with Acute Coronary Syndrome. Chinese General Practice Nursing 2019;17:2891-3.

19. Yang YJ, Lu Q, Zeng L. Effect of Cognitive Behavioral Intervention on Anxiety and Depression of Patients with Acute Coronary Syndrome. J Clin Res 2013;30:69-71.

20. Yu B. The effect of Beck's cognitive therapy on anxiety and depression in young and middle-aged patients with acute coronary syndrome. China Medical Herald 2017;14:170-2, 176.

21. Apolinário-Hagen J, Druge MFritsche L. Cognitive Behavioral Therapy, Mindfulness-Based Cognitive Therapy and Acceptance Commitment Therapy for Anxiety Disorders: Integrating Traditional with Digital Treatment Approaches. Adv Exp Med Biol 2020;1191:291-329.

22. Zhang XX, Zhou C, Duan HW, et al. Cognitive Behavioral Therapy for Relieving Depression or Anxiety in Parkinson's Disease: a Meta-analysis. Chinese General Practice 2019;22:2084-90.

23. Neufeld CB, Palma PC, Caetano KAS, et al. A randomized clinical trial of group and individual Cognitive-Behavioral Therapy approaches for Social Anxiety Disorder. Int J Clin Health Psychol 2020;20:29-37.

24. Yue M. Analysis of the application value of cognitive behavior intervention combined with preventive nursing in hospitalized patients with coronary heart disease. Psychological Monthly 2020;15:96.

25. Munyombwe T, Hall M, Dondo TB, et al. Quality of life trajectories in survivors of acute myocardial infarction: a national longitudinal study. Heart 2020;106:33-9.

Cite this article as: Yu H, Ma Y, Lei R, Xu D. A meta-analysis of clinical efficacy and quality of life of cognitive-behavioral therapy in acute coronary syndrome patients with anxiety and depression. Ann Palliat Med 2020;9(4):1886-1895. doi: 10.21037/apm-20-974 